CIUDAD Y TERRITORIO

ESTUDIOS TERRITORIALES

ISSN(P): 1133-4762; ISSN(E): 2659-3254

Vol. LII, № 204, verano 2020

Págs. $335-348$

https://doi.org/10.37230/CyTET.2020.204.10

CC BY-NC 4.0

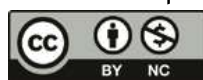

\title{
El acceso de los jóvenes al trabajo y la ciudad. Miradas territoriales de la desigualdad y la segregación en Santiago de Chile
}

\section{Luis Fuentes (1) \\ Sebastián RodríGuez-LEIVA ${ }^{(2) 1}$}

(1)Dr. en Arquitectura y Estudios Urbanos Instituto de Estudios Urbanos y Territoriales. Centro de Desarrollo Urbano Sustentable (2) Pontificia Universidad Católica de Chile MSc Gobernanza del Riesgo y Recursos Naturales, Ruprecht - Karls - Universität Heidelberg Centro de Desarrollo Urbano Sustentable. Pontificia Universidad Católica de Chile

RESUMEN: Chile ha sido unos de los países en el mundo en donde se ha aplicado con más ortodoxia el neoliberalismo. A partir de 1975, una serie de reformas en distintos ámbitos pusieron al mercado como el principal asignador de recursos. Con la llegada de la democracia, el modelo fue perfeccionándose y profundizándose a través de distintos mecanismos, siendo el más importante la apertura a los mercados globales. Esta inserción internacional del país a través de una economía nacional fuertemente globalizada ha impactado a su capital y sus habitantes, la cual ha sufrido procesos de reestructuración urbana, configurando un mercado laboral metropolitano y una geografía social segmentados, especialmente cuando analizamos los grupos más jóvenes y pobres de la población. Esto ha generado mecanismos de exclusión en las esferas más importantes de la sociabilidad urbana, que requieren ser desactivados por las políticas públicas.

PALABRAS CLAVE: Neoliberalismo; Santiago de Chile; Mercados del trabajo; Vivienda social.

Recibido: 20.10.2018; Revisado: 22.06.2019

Correo electrónico: Ifuentes@uc.cl NºRCID: https://orcid.org/0000-0003-1233-1563;

Correo electrónico: sirodri1@gmail.com No ORCID: https://orcid.org/0000-0002-7063-0177;

Los autores agradecen los comentarios y sugerencias realizados por los evaluadores anónimos, que han contribuido a mejorar y enriquecer el manuscrito original.

${ }_{1}$ Trabajo realizado en el contexto de los proyectos Fondecyt N 1190724 y Agencia Nacional de Investigación y Desarrollo, Proyecto

FONDAP N 15110020 


\title{
Young people's access to work and the city. Territorial looks of inequality and segregation in Santiago, Chile
}

\begin{abstract}
Chile has been one of the countries in the world where neoliberalism has been applied most orthodoxically. Since 1975 a series of reforms were established in various fields which set the market as the main allocator of resources. With the restoration of democracy, the model was improved and deepened through various mechanisms, global openness being the most important one. This international insertion of the country through a highly globalized national economy has impacted the capital city and its inhabitants, undergoing processes of urban restructuring and forming a metropolitan labour market and segmented social geography, especially for the younger and poorer segments of the population. This has generated mechanisms of exclusion in the most important areas of urban sociability, which need to be deactivated by public policies.
\end{abstract}

KEYWORDS: Neoliberalism; Chile; Labour markets; Youth unemployment; Urban inequality.

\section{Introducción}

as expectativas con las cuales se aplicaron las reformas neoliberales en América Latina aproximadamente desde los años ochenta, estaban basadas en un efecto gradual de crecimiento sostenido que generaría como consecuencia más empleo, mejores ingresos y una base más firme para la paz y el orden social luego de un período de ajuste (PORTES \& ROBERTS, 2005). Los cambios programáticos incluyeron una apertura unilateral al comercio exterior, privatización de las empresas públicas, nuevas formas de regulación de los mercados laborales, ajustes fiscales, reestructuración de los programas sociales y privatización de los fondos de pensiones, entre otras medidas.

Casi en su totalidad, los gobiernos de América Latina fueron aplicando con distinta intensidad tales postulados, formalizados más tarde en lo que se denominó el "Consenso de Washington", sobre la premisa de hacer sus países cada vez más atractivos para la inversión nacional y extranjera, y el desarrollo de los negocios internacionales. Esto, sumado a las tendencias globales de reestructuración productiva, desarrollo y masificación de las nuevas tecnologías de la información y comunicación, tuvo profundos efectos territoriales, particularmente en las grandes áreas metropolitanas, y también efectos sociales, especialmente en los segmentos de población joven y pobre.

En este marco, uno de los casos de mayor interés es el de Chile. Si bien corresponde a una economía pequeña, representando el $4,6 \%$ del Producto Interno Bruto (PIB) de América Latina y el Caribe en 2017 (BANCo Mundial, 2018), el ar- gumento detrás de esto se sustenta básicamente en tres factores. En primer lugar, las reformas en Chile fueron aplicadas de manera sistemática aproximadamente en 1975, lo que convierte a Chile en el primer país del mundo en donde se implementó un paquete de reformas que podríamos clasificar como "Neoliberal", incluso antes que en la Inglaterra de Thatcher (1979) y Estados Unidos de Reagan (1981). En segundo lugar, debido a que las reformas fueron aplicadas bajo un régimen dictatorial, la ortodoxia fue una de las características más importantes en términos de la intensidad en su implementación. En tercer lugar, Chile ha sido uno de los pocos países Latinoamericanos en donde los pilares del modelo se han mantenido estables en términos generales, ya que después de la recuperación de la democracia en 1990, los gobiernos en algunos casos incluso profundizaron aún más algunos aspectos.

Dicho esto, Chile adquiere gran relevancia y un caso de estudio interesante de análisis. El país se posicionó en un lugar atractivo para la inversión nacional e internacional, lo cual se reflejó en el lugar ocupado en los rankings sobre competitividad y libertad económica. Como consecuencia de esto, Santiago se transformó en una ciudad atractiva para los negocios (América Economía, 2004) y el ingreso per cápita aumentó considerablemente pasando de US\$5.000 en 1996 a casi US \$15.000 en 2014 según cifras del Banco Mundial.

Esto último podría vincularse a la hipótesis tradicional de la teoría económica, en que una economía competitiva contribuirá más a la calidad de vida, ya que un rápido crecimiento económico trae mayor demanda laboral, incrementa 
las oportunidades de empleo y disminuye el desempleo, la exclusión social y malas condiciones de vida; mientras el estancamiento y la declinación económica tiene impactos negativos en las relaciones sociales y la equidad, y por tanto afecta negativamente a los habitantes. Un elemento que evidencia claramente la relación entre una economía exitosa o competitiva y la sociedad, como sugieren FAINSTEIN (2001), GoRDON (2005) y TUROK (2006), se produce a través de los mercados del trabajo, los que transmiten los beneficios del desempeño económico a los distintos grupos sociales.

Sin embargo, estas hipótesis merecen ser rediscutidas y contrastadas con la evidencia empírica para el caso de las ciudades latinoamericanas y en particular, el caso chileno. En los últimos años el modelo ha entrado en fuerte cuestionamiento, al igual que en otros países, lo cual nos hace preguntarnos sobre los efectos territoriales y sociales de estas políticas. Considerando esto, analizaremos dos políticas que han tenido un especial interés social y territorial como lo son las reformas del mercado laboral y las políticas de vivienda social. Se asume que estas dos políticas han tenido un especial efecto sobre las personas (en especial en los más jóvenes) y sus capacidades para interactuar con otras personas en dos de las esferas de socialización más importantes en la ciudad: mercado del trabajo y el barrio. En las ciudades latinoamericanas y en especial en un régimen de libre mercado, la conexión entre estos dos aspectos es estrecho, ya que el salario condicionará fuertemente el lugar donde se puede vivir.

En este contexto, el presente artículo se propone explorar la siguiente pregunta: ¿Cómo las políticas en materia laboral y de vivienda social implementadas en Chile, incidieron en la capacidad de inclusión/exclusión en el mercado de trabajo y la residencia de los habitantes de Santiago, y particularmente, sus jóvenes?

Así, este trabajo analiza las relaciones entre políticas de libre mercado y los mecanismos que inciden en la capacidad de inclusión/exclusión de la población, haciendo énfasis en los más jóvenes. Para abordar esta problemática, se toma como base el aporte de KATZMAN (2009), quien plantea que las esferas más importantes de socialización de la ciudad tienen que ver con el mercado del trabajo, la distribución de la población en el territorio y los servicios públicos. Para este caso se considerarán las dos primeras esferas. A partir de la encuesta de hogares del Ministerio de Desarrollo Social, la Encuesta de Caracterización Socieconómica Nacional (CASEN), se extraen para diferentes años, desde 1987 a 2015, datos en tres dimensiones del empleo: a) participación del empleo. b) nivel de desocupados, 3) calidad del empleo. En cada una de estas dimensiones se analiza principalmente la edad, el ingreso y nivel educacional. Respecto a la distribución de la población en el territorio, se trabaja con información de los proyectos de vivienda social y de los jóvenes inactivos, considerando como fuente el Ministerio de Vivienda y Urbanismo de Chile (MINVU) y el censo del INE, respectivamente.

En cuanto a la estructura del documento, este se configura en cinco apartados, incluyendo el presente con fines introductorios. En el segundo apartado se presenta el contexto de Chile y Santiago y su evolución, junto con una breve reseña de las principales políticas aplicadas en las dos esferas analizadas. En el tercer apartado se presenta la evolución de los mercados del trabajo en Santiago sobre la base de algunos indicadores clave. En el cuarto apartado se analizan los principales cambios en la distribución de la población, producto de las políticas de vivienda social. Finalmente, en el quinto apartado se dan a conocer las principales reflexiones del trabajo en torno a los temas propuestos.

\section{Contexto nacional y evolución de Chile y Santiago}

En este trabajo, el neoliberalismo se entenderá según HARVEY (2009), como una teoría de prácticas político - económicas que afirma que la mejor manera de promover el bienestar del ser humano consiste en no restringir el libre desarrollo de las capacidades y de las libertades empresariales del individuo dentro de un marco institucional caracterizado por derechos de propiedad privada fuertes, mercados libres y libertad de comercio. BRENNER \& PECK \& THEODORE (2009), plantean la noción de "neoliberalización híbrida"2 para enfatizar el carácter constitutivamente incompleto, experimental y, finalmente, polimórfico de los procesos de neoliberalización, así como su carácter dependiente de la trayectoria de cada ola sucesiva de reestructuración regulatoria.

En Chile, este modelo de desarrollo se ha configurado como un proceso híbrido que ha pasado por distintas etapas y consensos, con avances y

2 Traducido de la expresión original: "Variegated neoliberalization”. 
retrocesos en diversos ámbitos de la economía y la vida social. Una primera etapa tiene lugar en los meses que siguieron al golpe militar de septiembre de 1973 , en donde se cambió radicalmente la política económica, abandonando de plano el antiguo modelo intervencionista y estableciendo un nuevo régimen que se formalizó aproximadamente en 1975 , con un paquete de medidas sustantivas que podrían calificarse como neoliberales. Con la vuelta a la democracia en 1990, no se evidenciaron grandes cambios en las políticas económicas. Por el contrario, los gobiernos de centro izquierda mantuvieron y en algunos casos profundizaron el modelo neoliberal. Un cambio relevante fue el aumento en los gastos sociales, el cual se financió con nuevos tributos y, gracias a ello, Chile siguió presentando un superávit significativo del sector público durante los noventa, equivalente al 1 y $2 \%$ del PIB, lo cual coincidió con uno de los períodos más extraordinarios de crecimiento económico jamás experimentado por este país, alcanzando el $7,7 \%$ anual como promedio entre los años 1990 y 1997. En la Fig. 1 puede observarse una panorámica de las reformas implementadas en Chile en distintos ámbitos.
Un análisis panorámico de estas reformas puede encontrarse en una amplia bibliografía que ha caracterizado este proceso ${ }^{3}$. Para el caso de este apartado se enfatiza en las reformas que afectaron principalmente dos de los ámbitos expuestos en la figura anterior: el mercado laboral y el desarrollo urbano. Pero ¿por qué es importante esto para Santiago? La ciudad capital fue uno de los lugares del país más impactados por este proceso de reestructuración de la economía, cambiando rápidamente su matriz productiva, desde una economía con un alto peso de la industria a una matriz prácticamente terciaria, cuestión relevante para la estructuración de un mercado laboral más flexible. Esto, sumado a la relevancia del sector inmobiliario y la valorización del suelo como activo, estrechó la relación entre la capacidad de pago y el lugar de residencia.

\subsection{Reformas al mercado laboral}

Desde la perspectiva neoliberal, antes de las reformas económicas la mayor parte de las legislaciones laborales en Latinoamérica se caracterizaban

\begin{tabular}{|c|c|c|c|c|c|}
\hline $\begin{array}{l}\text { Reforma } \\
\text { Fiscal }\end{array}$ & $\begin{array}{l}\text { Reforma } \\
\text { Comercial }\end{array}$ & $\begin{array}{l}\text { Reforma Mercado } \\
\text { Financiero }\end{array}$ & $\begin{array}{c}\text { Reforma del } \\
\text { Mercado Laboral }\end{array}$ & Privatización & $\begin{array}{c}\text { Desarrollo } \\
\text { Urbano }\end{array}$ \\
\hline $\begin{array}{l}\text { Reforma Fiscal, } \\
\text { 1975; presupuesto } \\
\text { equilibrado; } \\
\text { aumento de } \\
\text { los tipos fiscales; } \\
\text { en 1993, } \\
\text { modificación de las } \\
\text { leyes fiscales de } \\
\text { 1980. Auto } \\
\text { imposición de } \\
\text { compromisos } \\
\text { fiscales (balance } \\
\text { fiscal) desde } 2001 .\end{array}$ & $\begin{array}{l}\text { Gran reforma } \\
\text { en 1975-1979; } \\
\text { restricciones } \\
\text { cuantitativas } \\
\text { eliminadas; } \\
\text { imposición } \\
\text { de aranceles } \\
\text { uniformes de } \\
\text { importación } \\
\text { del } 11 \% \text {. } \\
\text { Facilitación } \\
\text { a la entrada } \\
\text { de capitales } \\
\text { extranjeros. } \\
\text { Firma de acuerdos } \\
\text { de libre comercio } \\
\text { con principales } \\
\text { mercados del } \\
\text { mundo. }\end{array}$ & $\begin{array}{l}\text { Gran reforma } \\
\text { en 1975-1979; } \\
\text { tipos de interés } \\
\text { liberalizados; } \\
\text { cuenta de } \\
\text { capitales semi- } \\
\text { abierta. Sistema } \\
\text { de pensiones } \\
\text { de capitalización } \\
\text { individual. } \\
\text { Adopción de tipos } \\
\text { de cambios } \\
\text { flotantes. Adopción } \\
\text { de los criterios } \\
\text { de la "corporate } \\
\text { governance". } \\
\text { Reformas } \\
\text { mercados de } \\
\text { capitales en } 1994, \\
2001,2007 \text { y } 2010 .\end{array}$ & $\begin{array}{l}\text { Liberalización } \\
\text { y flexibilización } \\
\text { del mercado } \\
\text { de trabajo (1979); } \\
\text { leyes laborales } \\
\text { reformadas } \\
\text { en } 1990 \text { que } \\
\text { no afectan } \\
\text { la estructura } \\
\text { de la regulación } \\
\text { laboral flexible. }\end{array}$ & $\begin{array}{l}\text { Importante, todas } \\
\text { las empresas } \\
\text { estatales vendidas } \\
\text { menos } 50 ; \\
\text { participación total } \\
\text { del sector privado } \\
\text { en los servicios } \\
\text { públicos a } \\
\text { excepción del agua } \\
\text { hasta } 1994 . \\
\text { Privatización } \\
\text { de últimas } \\
\text { participaciones } \\
\text { del sector público } \\
\text { en sanitarias } \\
\text { desde } 2010 .\end{array}$ & $\begin{array}{l}\text { Liberalización } \\
\text { del mercado de } \\
\text { suelos en } 1979 . \\
\text { Fomento a } \\
\text { las inversiones } \\
\text { inmobiliarias } \\
\text { mediante la } \\
\text { construcción de } \\
\text { viviendas sociales } \\
\text { a gran escala. } \\
\text { Desarrollo y fomento } \\
\text { a la infraestructura } \\
\text { urbana mediante } \\
\text { paternariado } \\
\text { público-privado. } \\
\text { Modernización } \\
\text { del sistema de } \\
\text { subsidios de } \\
\text { vivienda social. } \\
\text { Modernización } \\
\text { del sistema } \\
\text { de créditos } \\
\text { hipotecarios } \\
\text { (letras y mutuos), } \\
\text { la adopción } \\
\text { de regulaciones } \\
\text { sobre securitización" } \\
\text { y "leasing. }\end{array}$ \\
\hline
\end{tabular}

Panorámica de las reformas realizadas en Chile a partir de 1975 en distintas áreas.

Fuente: Extraído en parte de EDWARDS, 1997 y modificado por el autor.

${ }^{3}$ Edwards (1997) y (2001); Bulmer-Thomas (1994); CalCANo, (1989); Haggard \& Kaufman, (1992); Harvey (2007); Coloma
\& RoJAs (2000); CorTÁZAR (1993); FRENCH-DaVIS, (2008); LARRAin Y Vergara, (2001); Weller, (2000); entre otros. 
por constituir un mercado excesivamente rígido, donde no solo resultaba difícil introducir nuevas tecnologías o modificar la orientación de las firmas, sino que además los ajustes de mano de obra resultaban altamente complejos. Esto último, por los altos costos de los despidos, los engorrosos mecanismos para concretarlos y la existencia de monopolios en algunas actividades del mercado del trabajo (Coloma \& RoJAs, 2000). La justificación de tal situación, según EDWARDS (1997), es que en nuestra región históricamente se usó la legislación laboral como instrumento para alcanzar objetivos sociales.

Sin embargo, las reformas orientadas hacia un modelo de desarrollo económico de libre mercado obligaron a generar un mercado del trabajo compatible con las nuevas exigencias de dinamismo y flexibilidad, lo cual

"permitía reasignar recursos y permite que la economía responda rápidamente a los nuevos desafíos planteados por una mayor competencia exterior" (EDWARDS, 1997: 342).

Los supuestos -según el mismo economista-, es que liberar de estas distorsiones al mercado del trabajo permite tener efectos positivos en la distribución, porque estimula la expansión del empleo y aumenta los salarios en los segmentos más pobres de la sociedad.

Para el caso específico de Chile, las regulaciones relativas al mercado del trabajo pueden ser divididas básicamente en dos períodos. El primero de 1974 a 1991 tuvo como objetivo complementar el ajuste requerido por otras reformas estructurales e introducir flexibilidad en el mercado laboral con el fin de promover una mayor eficiencia y reducir las altas tasas de desempleo que se elevaron hasta niveles de dos dígitos. En particular, se avanzó en la abolición de algunos privilegios y licencias para desempeñar determinadas funciones, se cambió la legislación en materia de despido e indemnizaciones ${ }^{4}$, se derogó la afiliación obligatoria a los sindicatos y se limitó fuertemente su acción ${ }^{5}$, se cambiaron las reglas de la negociación colectiva y se prohibieron las huelgas $^{6}$. Adicionalmente en 1980 se modificó el sistema de pensiones ${ }^{7}$, que cambió un sistema de previsión social solidario manejado por el Estado por uno de capitalización individual administrada por el sector privado (Administradoras de Fondos de Pensiones, AFP). Esto fue acompañado por una privatización del sistema de salud mediante la seguritización de los trabajadores en entidades privadas conocidas como Isapre.

${ }^{4}$ D. L. 2.200 y Ley 18.372 de 1978 y 1981 respectivamente. ${ }^{5}$ D. L. 2.756 y D.L. 2.757 de 1979
En un segundo período, bajo los gobiernos democráticos a partir de 1991, se introdujeron nuevos cambios a la legislación laboral, los que contribuyeron a reforzar el poder negociador de los trabajadores, aunque de una manera coherente con el marco de mayor flexibilidad que se había impuesto en los años del gobierno militar (COLOMA \& RoJAs 2000). Al respecto, la legislación promulgada en 1991 mantiene la misma estructura anterior en cuanto a los tipos de sindicatos y a la afiliación voluntaria; sin embargo, se introducen modificaciones que disminuyen los requerimientos para formar sindicatos en empresas pequeñas, como también se reconoció la posibilidad de formar centrales sindicales (Coloma \& RoJAs, 2000). También se permiten la negociación multiempresa por acuerdo de las partes, es decir, se elimina la prohibición expresa que existía de negociar más allá de la empresa y se restablece el derecho a huelga, aunque con la posibilidad de que las empresas sustituyan sus trabajadores desde el primer día.

En las últimas décadas se han establecido nuevas reformas como la reducción de la jornada laboral de 48 a 45 horas a la semana, extensión de permisos en caso de nacimiento o defunción, homologación del ingreso mínimo legal al salario base, cotización del empleador para el seguro de cesantía, entre otras. También en fechas recientes se crearon los tribunales del trabajo con el objeto de agilizar y dar un juicio rápido a las controversias en materia laboral.

\subsection{Reformas en el desarrollo urbano y las políticas de vivienda social}

En materia de desarrollo urbano, las reformas también fueron importantes casi desde el comienzo de la instauración del nuevo modelo. Uno de los hitos más relevantes está dado por la promulgación de una nueva Política Nacional del Desarrollo Urbano (PNDU) en 1979, siendo la calificación del suelo urbano como un recurso "no escaso" una de las características más novedosas de dichas políticas. El objetivo fue eliminar las restricciones para el libre funcionamiento del mercado en términos del crecimiento natural de las ciudades.

"El uso del suelo queda definido por su mayor rentabilidad. La tierra es un recurso que se transa en forma libre (...) se definirán procedimientos y se eliminarán restricciones de modo de permitir el crecimiento natural de las áreas urbanas, siguiendo las tendencias del mercado" (Mınvu, 1981a: 10 y 13).

${ }^{6}$ D. L. 2.758 de 1979

${ }^{7}$ D. L. 3.500 y D.L. 3.501 en noviembre de 1980 
La inspiración para tal determinación estaba basada en la creencia de que intentar detener el crecimiento natural de las ciudades era un esfuerzo innecesario. DAHER (1989), recogiendo palabras de Harberger plantea que

"partiendo de la evidencia, yo tengo que concluir que crecimiento hacia afuera es un fenómeno económico natural, que los gobiernos no pueden parar aun cuando traten de hacerlo, lo que además es, seguramente, poco sabio intentar" (HARBERGER, 1979: 39).

Posteriormente en 1985, producto de los problemas de crecimiento inorgánico de las ciudades que estaba generando la desregulación, se creyó necesario proceder al ajuste o rectificación de la planificación empleada hasta ese entonces. Así, producto del trabajo de una comisión convocada por el Ministerio de Vivienda y Urbanismo, en 1983 se promulgó una nueva Política Nacional de Desarrollo Urbano (PNDU). Esta tenía cuatro declaraciones básicas: (a) el bien común y el rol del Estado, (b) el derecho a la propiedad (c) libre iniciativa y mercado, (d) la habitabilidad de los asentamientos humanos, (e) el carácter urbanístico de los asentamientos humanos. Esta política no tuvo el efecto esperado, principalmente debido a que no se promovió ninguna de las leyes que requería para operar como guía del desarrollo urbano y fue derogada en el año 2000. En la primera década de los noventa hubo algunos intentos por avanzar en una política de desarrollo territorial, la cual no fue aprobada oficialmente.

Posteriormente a la derogación de la PNDU de 1985, en el año 2001 se creó un grupo de trabajo para la reforma urbana,

"el objetivo sería hacer el levantamiento de las principales reformas que requería el Estado para abordar los desafíos urbanos del nuevo siglo, tanto respecto a la institucionalidad como a los instrumentos públicos necesarios para el mejoramiento de las ciudades" (Minvu-PNUD, 2012: 96).

La mirada estaba puesta en la celebración del Bicentenario del país (2010), teniendo a las ciudades como el escenario más importante de celebración de dicha importante festividad nacional. Los cuatro subgrupos de trabajo indican las prioridades que tendrían los temas a evaluar: (a) movilización de recursos y financiamiento para el desarrollo urbano, (b) uso del suelo, equipamiento y vivienda, (c) instrumentos de planificación y gestión urbana y territorial, y (d) participación, espacios públicos y cultura. De cierta manera, esta reforma urbana promovía en su espíritu reformular el rol del Estado en el desarrollo urbano de las ciudades, sin embargo, no tuvo un efecto muy relevante en este sentido, principalmente porque no hubo reformas estructurales en la institucionalidad ni en las leyes.
Posteriormente, en el 2009 se generaron esfuerzos por la generación de una nueva PNDU, específicamente en este año se generó un trabajo del Ministerio de Vivienda y Urbanismo con el apoyo del Programa de Naciones Unidas para el Desarrollo (PNUD), lo cual se materializó en un documento que se denominó "Hacia una política de Desarrollo Urbano: Ciudades Sustentables". Finalmente, en 2014 se promulga una nueva PNDU después de un esfuerzo de dos años de trabajo de una Comisión Asesora Presidencial, que se pretende que guíe el desarrollo futuro de las ciudades y centros poblados de Chile.

Más allá de estos esfuerzos por generar una PNDU, una de las políticas públicas que mayor impacto tuvieron en el desarrollo urbano de nuestras ciudades fue la Política de Vivienda Social (PVS). Según HIDALGo (1999), las principales transformaciones pasaron por la creación de los comités habitacionales comunales en 1974, concibiendo la vivienda como un derecho, pero producto del esfuerzo y el ahorro de las familias, en el contexto de un mercado abierto de viviendas en donde los privados tienen un papel muy relevante en términos de oferta. Como herramienta para apoyar este proceso se creó un eficiente sistema de subsidio habitacional que generó una creciente demanda de viviendas sociales para distintos segmentos sociales que fueron beneficiados por estas políticas.

Bajo este enfoque, el nuevo sentido de la habitación social sugiere que la vivienda es un objeto al cual se adquiere ahorrando, y no lo concibe como un derecho natural e inherente a las necesidades de desarrollo familiar, siendo el mercado quien asigna los recursos y no el Estado, este último relega su accionar a la subsidiariedad, la gestación de regulaciones y fomento a la iniciativa privada, no así a la producción de viviendas por medio de sus instituciones (RIVERA, 2012).

Un catastro elaborado por la Secretaría Ministerial de Vivienda y Urbanismo de la Región Metropolitana, detectó más de 294 asentamientos irregulares que servían de habitación para más de 44 mil familias y casi 223 mil personas. HIDALGO (1999), plantea que solo entre 1979 y 1986 se erradicaron más del $60 \%$ de las familias que habitaba campamentos, las que accedieron a viviendas sociales nuevas en la periferia de la ciudad, en municipios que no contaban con infraestructura ni medios como para recibir a estos nuevos habitantes, consolidando una ciudad con una estructura polarizada. El nuevo sistema de subsidios habitacionales fue muy eficiente en términos cuantitativos, ya que solo entre 1978 y 1995 se construyeron casi medio millón de viviendas.

La llegada de la democracia a Chile no generó un cambio sustancial en las políticas de vivienda 
social, sino más bien todo lo contrario. El modelo de postulación y de construcción de viviendas bajo un esquema de mercado se potenció aún más construyéndose casi 100 mil viviendas anuales, mal localizadas y con especificaciones técnicas muy deficientes y rompiendo las redes sociales de los habitantes, dado la imposibilidad de elegir donde vivir.

Posteriormente, después del 2000 ha habido cambios en la política, lo cual de cierta manera reconoció los graves problemas que generó la masiva construcción periférica de soluciones habitacionales, incentivando la segregación y los problemas sociales en comunas pobres de las ciudades chilenas.

\section{Segmentación de los mercados de trabajo metropolitano en Santiago}

Se ha planteado en la literatura que la importancia del trabajo radica en que este, a) permite a las personas ganarse el sustento y tener seguridad económica, vital para lograr un crecimiento económico equitativo, la reducción de la pobreza y la igualdad de género; b) permite a los ciudadanos participar plenamente en la sociedad y les confiere un sentido de dignidad y valía personal; c) contribuye al interés público, donde el cuidado de otras personas fomenta la cohesión y crea vínculos en las familias y las comunidades; y d) consolida a las sociedades (PNUD, 2015: 1). Considerando el rol relevante que ejerce el trabajo en las personas, a continuación, se pretende caracterizar la evolución de los mercados metropolitanos del trabajo en Santiago y el efecto de las reformas implementadas.

Un primer elemento por considerar es que en la Región Metropolitana entre los años 1982 y 2013 , la tasa media de crecimiento de la Población Económicamente Activa (PEA) ha estado por sobre la tasa de población total. El crecimiento medio de la PEA ha tenido un incremento en torno al $2,8 \%$, mientras que la tasa de población ha disminuido desde $1,9 \%$ a $1,5 \%$ en el último período intercensal. Esto implica que la oferta en el mercado del trabajo ha estado creciendo a una tasa mayor que la población total.

En esta misma línea, la tasa media de crecimiento de los ocupados se ha comportado en la misma proporción que la de la PEA, a excepción del período 1982 - 1992 donde creció casi al doble. Una interpretación de estas cifras estaría relacionada con que el impulso inicial de las reformas tuvo un efecto importante en la relación con el aumento del empleo, mientras que los períodos sucesivos no tuvieron el mismo nivel de impacto.

En la FIG. 2 se muestra la tasa de desempleo en la Región Metropolitana, tasa que podría definirse como el balance entre la oferta y la demanda de empleo. Al respecto, se puede observar cómo en el período analizado, el promedio de la tasa de desempleo fue de un 7,9\%. Asimismo, a lo largo de este período, la tasa de desempleo en la región mostró valores que en general, se encuentran por debajo del $10 \%$, salvo algunas excepciones correspondientes a fases de crisis económicas globales, debido a la apertura de la economía a los shocks internacionales como lo fueron en los casos de la crisis asiática después de 1998 o la crisis subprime en el 2008 y parte del año 2009.

Sin embargo, al tener una segunda visión de las cifras de desempleo, es posible constatar que éstas esconden importantes segmentaciones en términos de edad, ingresos y educación. Cabe mencionar, que el término "segmentación" que se utiliza en este caso, no solo implica una diferenciación, sino también, la existencia de barreras para el tránsito de una a otra categoría (KATZMAn, 2009). Siguiendo esta lógica, WELLER (2011) plantea que las reformas recientes aplicadas al mercado del trabajo han traído consigo cuatro formas de exclusión: la exclusión del mercado laboral, del empleo, del empleo productivo y del empleo de buena calidad.

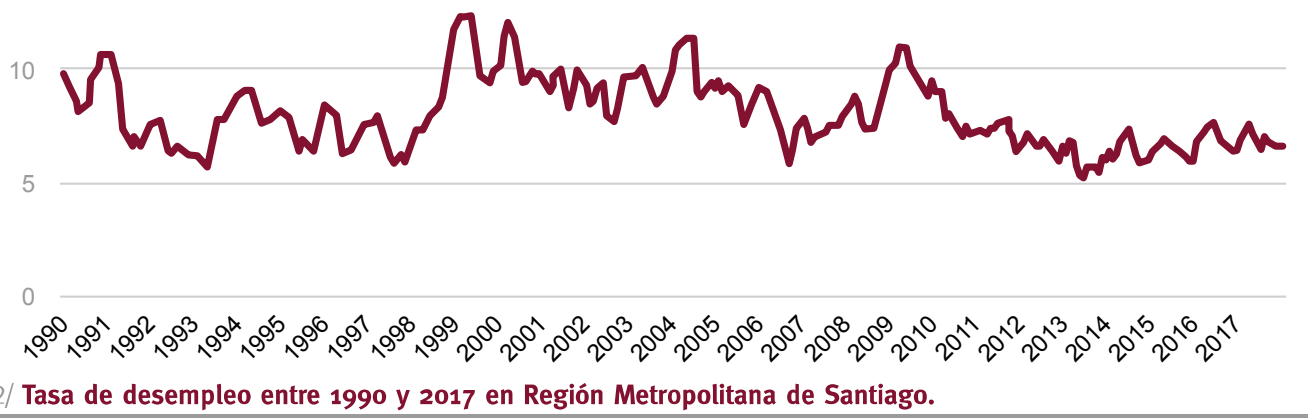

Fuente: InStituto Nacional de Estadisticas. 


\begin{tabular}{lcccccc}
\hline $\begin{array}{c}\text { Grupos } \\
\text { de edad }\end{array}$ & $\mathbf{1 9 8 7}$ & $\mathbf{1 9 9 0}$ & $\mathbf{2 0 0 0}$ & $\mathbf{2 0 1 7}$ & $\begin{array}{c}\text { Variación } \\
\mathbf{1 9 8 7 - 2 0 1 7}\end{array}$ \\
\hline $\mathbf{1 5}$ a 19 años & 17,2 & 17,8 & 16,0 & 10,3 & $-6,9$ \\
\hline $\mathbf{2 0}$ a $\mathbf{2 4}$ años & 60,8 & 59,5 & 56,5 & 46,9 & $-13,9$ \\
\hline $\mathbf{2 5}$ a 34 años & 70,6 & 71,6 & 77,1 & 75,5 & 4,9 \\
\hline 35 a 44 años & 70,8 & 72,7 & 77,9 & 77,6 & 6,8 \\
\hline $\mathbf{4 5}$ a 54 años & 62,3 & 66,4 & 76,3 & 76,0 & 13,7 \\
\hline 55 a 64 años & 43,1 & 46,8 & 56,3 & 64,4 & 21,3 \\
\hline Promedio & 53,0 & 55,3 & 60,1 & 64,2 & 11,2 \\
\hline
\end{tabular}

FIG. 3/ Tasas de participación en el mercado del trabajo por grupos de edad en la RMS.

Fuente: Elaboración propia a partir de Encuesta CASEN, diversos años.

En este sentido, es que, al observar las tasas de participación por grupos de edad en Santiago, se puede argumentar que existen diferencias relevantes no solo por segmento etario, sino también en el tiempo analizado. En específico, los grupos de población joven muestran: a) una tasa de participación menor que el resto de los grupos; b) una variación negativa en el tiempo, es decir, la tasa va disminuyendo en el período analizado; c) en particular, el grupo de edad entre 20 y 24 años muestra la mayor variación negativa, lo que se contrapone a la variación que muestra el grupo entre 55 a 64 años, con el mayor nivel de participación.

Una situación similar, en términos de segmentaciones, se produce cuando se analiza la participación en el mercado laboral de los distintos sectores sociales estratificados por ingresos. Mientras el decil más pobre en el año 2017 no superaba el $29 \%$ de participación, el decil más rico llegaba al $75,7 \%$ de participación en el mercado del trabajo. En cuanto a las tendencias, estas barreras incluso han aumentado desde 1990 a la actualidad. Al respecto los datos de CASEN, muestran que la participación del primer decil disminuyó en $15 \%$, pasando de $44,3 \%$ a $29 \%$; mientras que la del último decil aumentó más de $13 \%$, pasando de $62,3 \%$ a $75,7 \%$. Respecto a la segmentación por niveles educacionales, cabe señalar que los datos de la misma encuesta si bien muestran diferencias importantes en la participación en el mercado laboral, su evolución entre 1990 y 2009 no es tan relevante.

Otro tipo de exclusión guarda relación con las reformas orientadas hacia la flexibilización de las relaciones laborales, las que aumentaron las barreras de acceso al empleo. Por ejemplo, para el caso de la Región Metropolitana de Santiago no se observan diferencias muy importantes en el desempleo entre hombres o mujeres, aunque se debe reconocer que entre 1990 y 2009 siem- pre el desempleo femenino supera al masculino. Las diferencias más importantes se producen por edad, tal como se puede apreciar en el cuadro que se muestra a continuación (FIG. 3). Los datos de la encuesta CASEN, nos muestran los altos niveles de desempleo en los jóvenes, los cuales duplican y en algunos años triplican las tasas de desempleo promedio de la RMS (Región Metropolitana de Santiago).

Desde los 25 años en adelante, las tasas de desempleo están en torno al promedio o más bajas, y el corte de edad con menor desempleo es entre los 45 a 54 años (FIG. 4). Otro tema relevante que demuestra la existencia de barreras de entrada al empleo es la evolución de los distintos grupos. Como se observa en el cuadro prácticamente todos los grupos aumentan su desempleo en períodos de crisis. Asimismo, los grupos de población joven (15 a 24 años) son los únicos segmentos que superan al promedio; el resto se encuentra muy por debajo.

Si cambiamos la variable de edad por el nivel de ingresos, nos encontramos con un panorama similar en cuanto a las desigualdades en el acceso al empleo. Al respecto, el nivel de desempleo de los deciles más pobres supera ampliamente al resto, llegando a niveles en donde más de un tercio de los trabajadores se encuentran en paro. De esta manera, se genera una relación en la cual a medida que aumenta el nivel de ingreso, disminuye proporcionalmente la tasa de desempleo, llegando a niveles promedio de desempleo prácticamente inexistentes en el decil más rico. En cuanto a la variación temporal de esta tendencia desde 1987 hasta la actualidad, los datos muestran que las barreras de entrada al empleo se han ido manteniendo constantes. Es interesante que, la mayor brecha se produce en los tres primeros deciles con el resto, aunque el primer decil es notablemente el más afectado por el desempleo.

Respecto a la exclusión asociada al acceso al empleo de calidad, en el caso de Santiago, los

\begin{tabular}{|lrrrc}
$\begin{array}{c}\text { Grupos } \\
\text { de edad }\end{array}$ & $\mathbf{1 9 8 7}$ & $\mathbf{1 9 9 0}$ & $\mathbf{2 0 0 0}$ & $\begin{array}{c}\text { Variación } \\
\mathbf{1 9 8 7 - 2 0 1 7}\end{array}$ \\
\hline $\mathbf{1 5}$ a 19 años & 30,9 & 27,2 & 28,6 & 27,9 \\
\hline $\mathbf{2 0}$ A 24 años & 17,9 & 18,7 & 18,8 & 15,8 \\
\hline $\mathbf{2 5}$ a 34 años & 8,0 & 9,7 & 8,5 & 7,8 \\
\hline 35 a 44 años & 4,6 & 7,6 & 5,5 & 5,4 \\
\hline $\mathbf{4 5}$ a 54 años & 5,0 & 7,8 & 4,6 & 5,1 \\
\hline 55 a 64 años & 6,4 & 7,3 & 4,4 & 5,1 \\
\hline Promedio & 9,4 & 13,1 & 7,7 & 9,8 \\
\hline
\end{tabular}

/ Tasas de desocupación por grupos de edad en la RMS.

Fuente: Elaboración propia a partir de Encuesta CASEN, diversos años. 
datos de la encuesta CASEN muestran que, a pesar de que el promedio de asalariados sin contrato ha disminuido lentamente entre 1990 y 2017 , de un $15,2 \%$ a un $10,9 \%$, se puede sostener el argumento de que en general, el empleo precario se ha expandido. Esto se sustenta en las diferencias de género, donde las mujeres asalariadas sin contrato muestran en promedio un nivel superior al de los hombres a lo largo del período analizado. Asimismo, si analizamos los datos considerando los grupos etarios, el grupo donde se evidencia la mayor magnitud de empleo desprotegido ha sido el de los más jóvenes (15 a 19 años) en donde en 1990 un 27,1\% trabajaba sin contrato y en el año 2015 alcanzó el 26,8\%, siendo el grupo más perjudicado, ya que el resto de los grupos se encuentran en torno al promedio o por debajo de él. Ahora, si analizamos la misma variable, pero cruzada por deciles de ingresos, nos encontramos con un panorama similar, ya que existe una relación proporcional entre pobreza y acceso al trabajo sin contrato. En general, se observa que en todos los deciles crece el empleo desprotegido, pero la proporción de asalariados del decil más pobre supera ampliamente al decil más rico. Además, el decil en el que más crece el empleo desprotegido es en el decil más pobre. No obstante, la brecha respecto al resto no es tan amplia como los casos anteriores.

Finalmente, si bien en Chile el empleo informal es uno de los más bajos de Latinoamérica, las cifras realizadas por distintos estudios plantean que no es un tema poco relevante ya que, por ejemplo, según las cifras de la Comisión Económica para América Latina y el Caribe (CEPAL) habría bajado desde 38 a 30\% entre 1990 a 2009. Estas cifras sin duda son más altas que las que existían en 1978 antes de las reformas laborales, las que alcanzaban el 25,3\% (FueNTES \& MENESES, 1987). Esto es preocupante, ya que, en este caso a la desprotección laboral, se suma la desprotección en materia de salud y previsión social, lo cual implica un aumento en el grado de riesgo e inseguridad para un número importante de familias. También algunos estudios realizados han puesto el acento en el alto nivel de fragilidad en la duración del empleo. Por ejemplo, según cifras del Instituto Nacional de Estadísticas se estima que el $41 \%$ de los empleos en el sector privado dura menos de un año.

\section{Reestructuración socio-espacial en Santiago}

La distribución de la población en el territorio es un atributo de las ciudades, toda vez que el nivel de segregación social puede utilizarse para caracterizar la estructura social urbana, lo que permite hacer juicios sobre la naturaleza más o menos segregada de una determinada urbe. La segregación residencial urbana disminuye las oportunidades de movilidad social en las clases sociales menos privilegiadas, pues reduce sus redes de contactos y su capital social en el campo laboral; también estrecha sus posibilidades de alcanzar una educación de calidad, la socialización en torno a valores normativos funcionales al mundo del trabajo y la expansión de su capital cultural; $y$, en general, restringe su posibilidad de acceder a mejores empleos, debido a los costos de transporte, las discriminaciones generadas por el lugar de residencia y otros factores (Katzman \& Retamoso, 2005; Rodríguez \& ArriaGADA, 2004; SABATINI \& CÁCERES \& CERDA, 2001). Según PNUD (2014) es necesario abordar la segregación residencial como política del Estado, ya que mejorar el transporte y reducir los costes de conseguir mejores empleos integrará los mercados laborales y mejorará el acceso a las oportunidades de empleo.

En general las ciudades latinoamericanas se han caracterizado por sus altos niveles de segregación y desigualdad. De hecho, se ha utilizado la expresión "conos de altos ingresos", para designar la concentración espacial de los grupos de elite económica en ciertos sectores de la ciudad que asume aquella forma geográfica de cono (CEPAL, 2014), lo cual implica un alto nivel de segregación de estos sectores sociales en el espacio.

Para el caso de Santiago, todas las aproximaciones e independientemente de la escala de análisis, destacan la mayor presencia de grupos altos en el tradicional cono de alta renta de Santiago y la existencia de importantes áreas de concentración de los grupos más bajos en la periferia, principalmente en algunas comunas localizadas en el sur de la ciudad (LINK \& VALENZUELA \& FUENTES, 2015; Agostini \& al., 2016). Al respecto, ARRIAGADA (2010) argumentó que la segregación en Chile había aumentado producto de la incidencia de la política de vivienda social en algunas zonas de la ciudad y de la concentración de infraestructura urbana en zonas acomodadas del sector oriente, lo cual se suma a la expansión vertiginosa hacia la periferia subequipada (FIG. 5).

En este mismo sentido, es que, SABATINI (2000) planteó que la liberalización de los mercados urbanos en el país, y particularmente en Santiago, provocó la erradicación de asentamientos precarios que habían surgido previo a 1973 y que, luego del golpe de Estado, se buscó "limpiar socialmente" las zonas donde estos se localizaban y que correspondían a áreas de altos ingresos, 


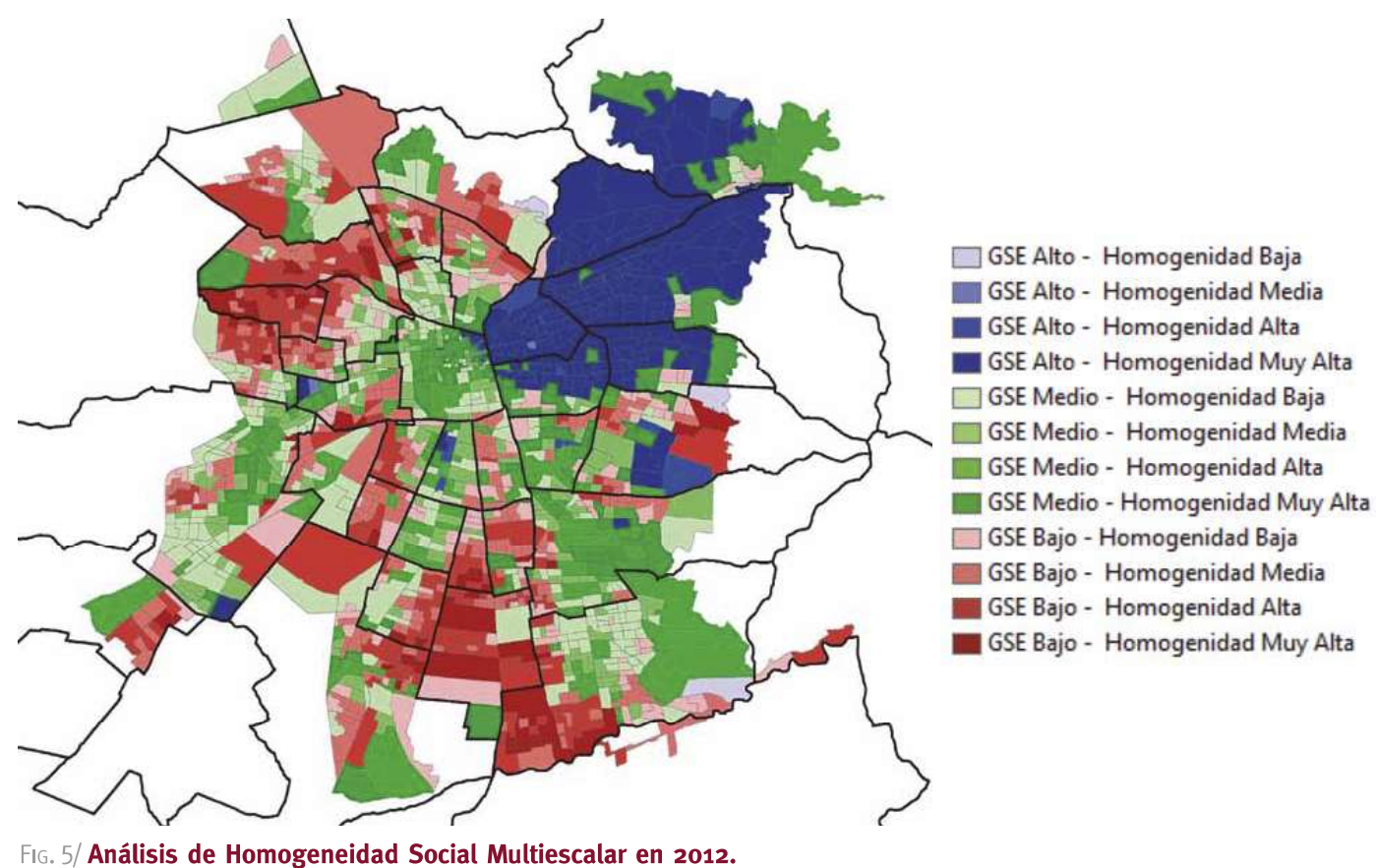

Fuente: ObSERVATORIO de CIUdAdes UC.
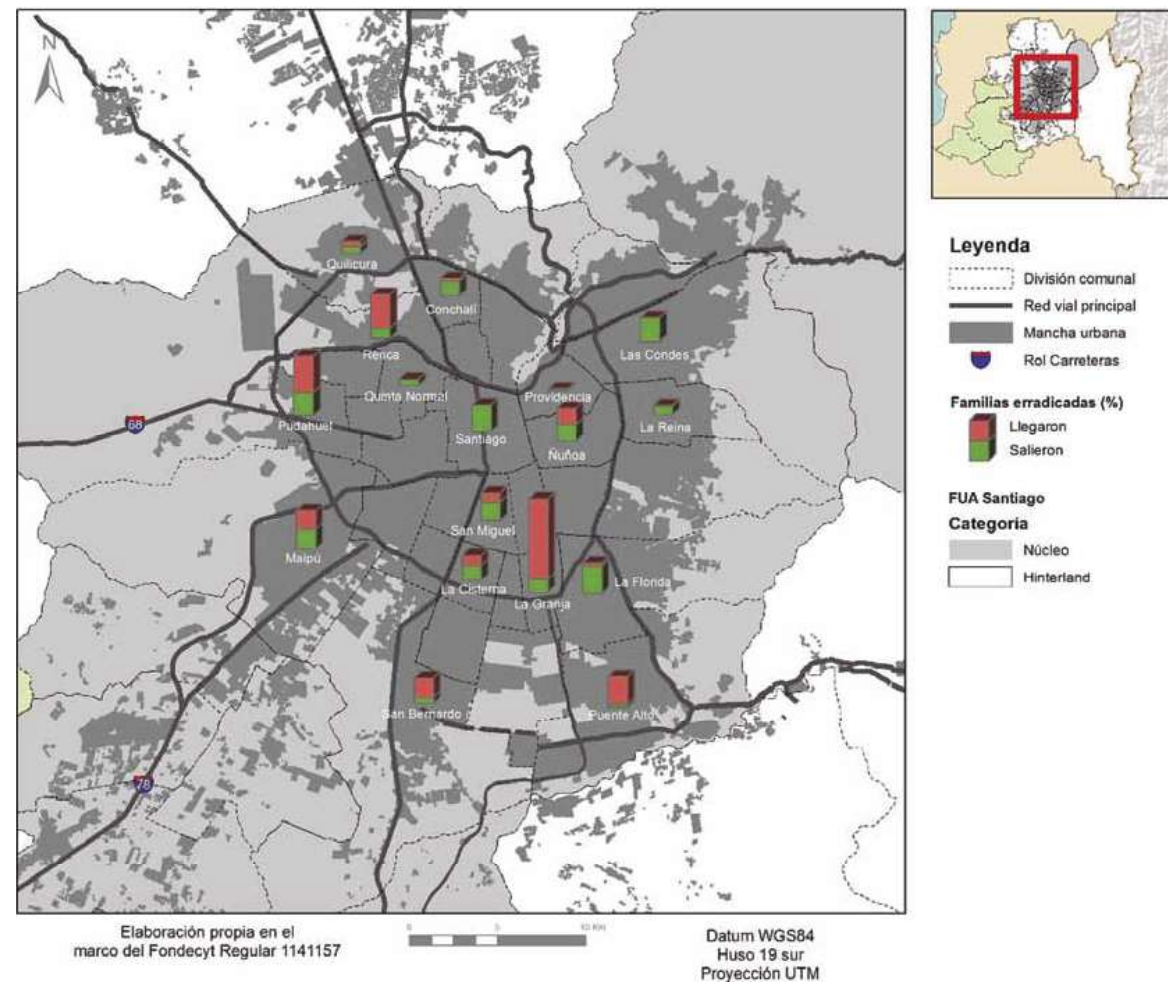

FIG. 6/ Familias erradicadas entre 1979 y 1985 de acuerdo con la municipalidad de radicación y erradicación.

Fuente: Elaboración propia en base a información de HIDALGO, (2004). 
dando paso al actuar del agente inmobiliario privado (SABATINI, 2000: 50). Así, una de las políticas que más ha incidido en la estructura social actual de la ciudad ha sido la política de vivienda social y la erradicación de asentamientos irregulares (ARRIAGADA, 2010). Hacia 1979, los campamentos se encontraban distribuidos geográficamente de manera relativamente homogénea en la ciudad (HIDALGO, 2004). Solo entre 1979 y 1985, se trasladaron casi 180.000 personas en la ciudad desde los campamentos a sus nuevas viviendas, es decir casi un $5 \%$ de la población de la región. El problema es que, como se observa en el mapa (FIG. 6), algunas comunas de la ciudad se transformaron en receptoras netas de estas viviendas, mientras otras comunas (sobre todo el sector este de la ciudad) se transformaron en expulsoras netas. Esto no hizo más que potenciar esta estructura social, ya que buena parte de las viviendas sociales (36\% del total construido entre 1978 y 1995) se levantaron en tres comunas de la ciudad, todas ellas ubicadas en el sur. Por el contrario, entre 1979 y 1992 en el este de la ciudad, es decir, comunas como Santiago, Las Condes o Providencia, no se construyó ninguna vivienda social (HIDALGO, 1996).

En esta misma lógica, si se analiza la distribución espacio temporal de los proyectos de vivienda social en la RMS, se puede apreciar que estos han ido trasladándose de forma constante en el tiempo, hacia la periferia de la ciudad, específicamente en los sectores surponiente y norponiente del área urbana de Santiago (Ver FIG. 7).

Las consecuencias de este proceso han sido caracterizadas como "el lado oscuro de la política de vivienda en Chile" (Duccl, 1997) o "el problema de los con techo" (RodRígueZ \& SUGRANYES, 2004), ya que más allá de solucionar el problema de habitabilidad ha potenciado la malignidad de la segregación generando el efecto ghetto (SABATINI \& CÁceres \& CERdA, 2001). Según SierRalta (2008), los fenómenos de desintegración social más frecuentemente enunciados por los investigadores en este campo son el desempleo e inestabilidad laboral, el alcoholismo y la drogadicción en los adultos; y el retraso escolar, malos resultados escolares y deserción de la escuela, maternidad adolescente, desempleo e inactividad juvenil. Esta hipótesis puesta a prueba para el caso de Santiago ha sido especialmente interesante. SIERRALTA (2008), plantea que, respecto a los resultados arrojados por los análisis de regresión entre segregación e inactividad juvenil de pobres, hay elementos destacables que tienen que ver con la evidencia para sostener que la segregación impacta fuertemente en las tasas de inactividad juvenil y también la existencia de una profunda brecha de género entre estas tasas.

En el mapa de la FIG. 8 se puede observar esta relación entre los proyectos de vivienda social y la

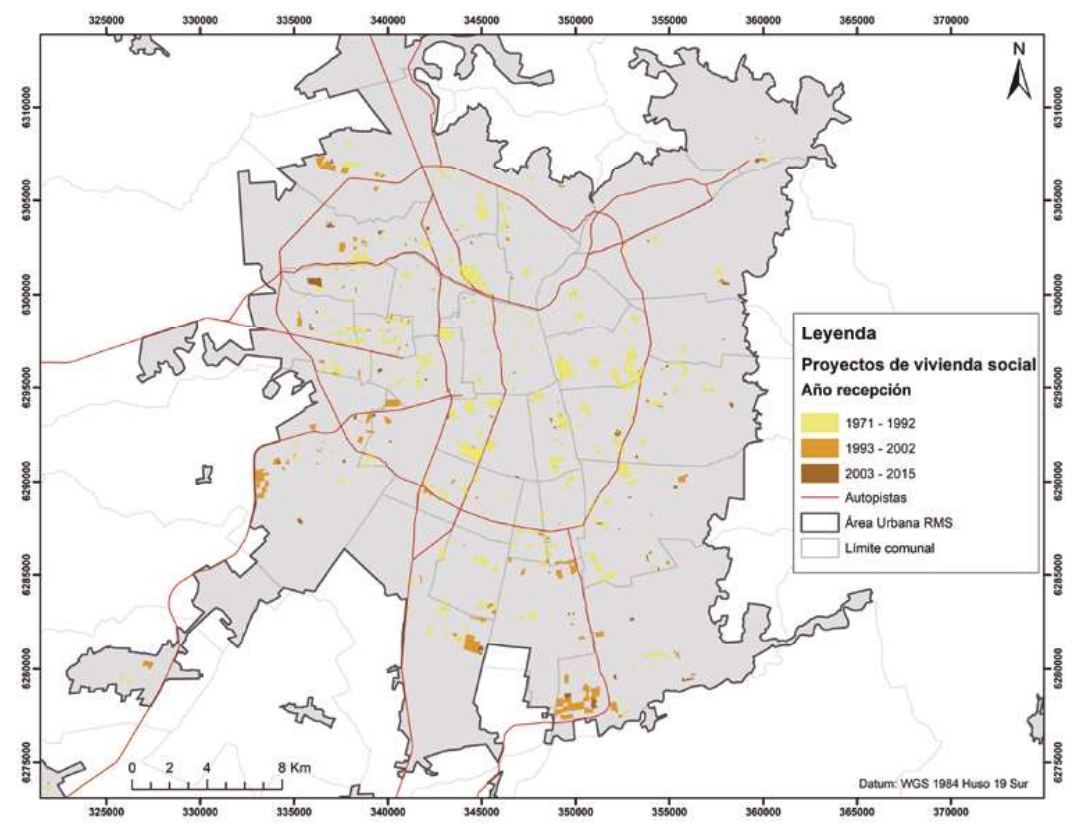

Evolución espacio temporal de los proyectos de vivienda social en la RMS.

Fuente: Elaboración propia con base en Catastro Nacional de Condominios Sociales realizado por el MINISTERIO DE VIVIENDA y URBANISMO, 2015. 


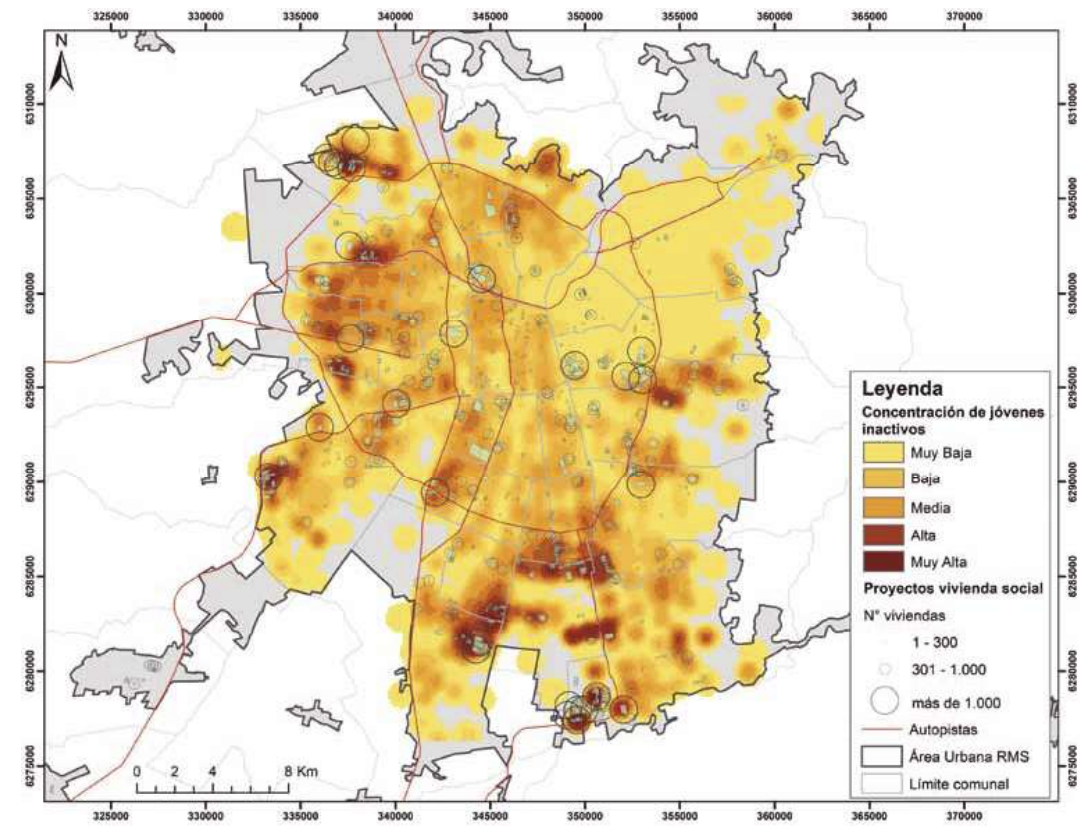

FIG. 8/ Sobreposición de los proyectos de vivienda social construidos entre 1992 y 2015 en Santiago y densidad de jóvenes inactivos por zonas censales en 2017.

Fuente:Elaboración propia en base a información de CIS Techo y Levantamiento Censal del 2017.

densidad de jóvenes en situación de inactividad. Se puede observar que en muchos casos hay un alto nivel de correlación espacial, sobre todo en los sectores más periféricos como en la zona norte en la comuna de Quilicura y en el sur en los casos de las comunas de San Bernardo, La Pintana y Puente Alto. Sin embargo, esta correlación no puede determinar causali-dad entre estas dos variables, sino más bien solo una coincidencia espacial.

A pesar de esto, esta relación demuestra la conexión y relación entre el mercado del trabajo y el lugar de residencia y el incremento del aislamiento de dos de las esferas de socialización más importantes en la ciudad. La relevancia de todo esto las podemos encontrar en palabras de KAZTMAN (2009: 40), quien señala que

"la frecuencia y la calidad de los contactos entre miembros de distintas clases es directamente proporcional a la disposición a colaborar entre sí y a la exposición a oportunidades para la construcción de patrones de negociación de conflictos. Por el contrario, el aislamiento físico incrementa las probabilidades que cada clase elabore perfiles imaginarios de las otras con independencia de las virtudes intrínsecas de sus miembros. Cuanto mayor el nivel de aislamiento entre las clases y su extensión temporal, más rígidos serán los estereotipos mutuos y las dificultades para su modificación".

\section{Discusión final}

Los datos muestran que procesos estructurales han jugado un papel muy relevante en la transformación urbana de Santiago, reproduciendo formas de exclusión social a través del mercado laboral y barreras espaciales. Así, la desintegración social pasa a constituir el elemento fundamental de (des)construcción de los espacios metropolitanos. Las reformas en los mercados del trabajo y las políticas de desarrollo urbano (al menos las analizadas) han tendido a segmentar la población y a generar barreras que dificultan la integración de la sociedad, elementos que se sobreponen, intensificando la exclusión social.

A partir del análisis del mercado del trabajo se pueden concluir tres aspectos. En primer lugar, que el crecimiento económico generado no necesariamente ha contribuido a aumentar las tasas de participación laboral, las cuales se mantienen bajas sobre todo en los jóvenes y en los sectores de menores ingresos. Lo segundo, es que se están produciendo importantes segmentaciones en el acceso al empleo, que se manifiestan en tasas de desempleo muy altas sobre todo en jóvenes y sectores de menores ingresos. Finalmente, lo tercero es que está aumentando crecientemente el empleo desprotegido o de baja calidad, que se manifiesta en la desprotección 
vía contratos, en la alta rotación laboral y en el aumento de la informalidad.

Respecto a la distribución de la población, la política de vivienda social contribuyó a la construcción de la estructura social actual de la ciudad, en donde la segmentación de los espacios es un elemento muy relevante. Esto complejiza cualquier tipo de políticas, dado que la segregación social ha incentivado una estructura de acceso a servicios muy desigual, lo cual se suma al desigual acceso al mercado laboral.

Como conclusión general se puede plantear que, el proceso de reformas iniciado tempranamente en Chile supone el traspaso de los riesgos y las responsabilidades sobre el bienestar a los individuos, y justamente quienes establecen los vínculos más débiles con el mercado del trabajo (es decir jóvenes y pobres) y además quedan relegados en ciertos sectores de la ciudad, son quienes resultan más perjudicados. La disminución de los recursos personales para interactuar en las esferas más importantes de la socialización en la ciudad como lo son el trabajo y el barrio genera un clima en el cual los lazos con el resto de la sociedad se rompen y difícilmente podrán volver a ser atados, ya que la segmentación social tiende a reproducirse, dificultando la construcción cohesionada de la ciudad. Estas políticas en general han tendido a aumentar las vulnerabilidades, entendidas como la posibilidad de deteriorar los logros del ámbito del desarrollo humano y su sostenibilidad (PNUD, 2014) de las personas afectadas a estos mecanismos de exclusión en el ámbito del acceso al trabajo y su localización residencial.

Estos mecanismos de exclusión están contribuyendo a mayores amenazas a la convivencia, en donde el miedo y las desconfianzas se consolidan como fuertes condicionantes de la interacción social. Estas amenazas se fundan, dentro del contexto que KATZMAN (2009) ha descrito como específico de este período, en el aumento de las expectativas de las personas ante los favorables escenarios políticos y económicos que se vivían hasta hace pocos años. Las altas tasas de crecimiento económico, el aumento en la cobertura educacional, la consolidación de la democracia y los discursos políticos, han alimentado las esperanzas de crecientes cantidades de población históricamente relegadas, de mejorar y alcanzar una posición social más igualitaria, con pleno acceso al bienestar y calidad de vida. Sin embargo, la realidad choca con la esperanza de ejercer el pleno derecho de la ciudadanía, dadas las crecientes debilidades que se producen en el vínculo entre los sectores más bajos de la pirámide social y el mercado del trabajo, a lo que se debe sumar el relegamiento y aislamien- to espacial, sobre todo en los jóvenes y pobres urbanos.

El espacio de política pública nacional destinado a mejorar las capacidades de lidiar con circunstancias adversas se ve limitado cada vez más conforme la globalización se profundiza. (PNUD, 2014:2). Por tanto, es necesario hacer cambios de enfoque en las políticas que afectan estas segmentaciones y erosionar estos mecanismos de exclusión. Redirigir esfuerzos hacia políticas públicas en materia de mercado de suelo e integración social; y en materia laboral, hacia la protección de los derechos sociales y aumentar la protección laboral, parecen ser el horizonte que se debe seguir.

\section{Bibliografía}

Agostinl, C. \& al. (2016): "Segregación residencial de ingresos en el Gran Santiago, 1992 - 2002: una estimación robusta". EURE, (127) 42; 159 - 184.

ARRIAGAdA Luco, C. (2010): Segregación residencial según dos modelos de urbanización y bienestar: estudio comparado de las áreas metropolitanas del Gran Santiago, Toronto y Vancouver. Notas de Población 91. CEPAL.

Banco Mundial (2018): "Datos Banco Mundial. Recuperado el 12 de agosto de 2018" desde: https://datos.bancomundial.org/indicador/NY.GDP. MKTP.CD?locations $=$ CL-ZJ

BuLMER-THOMAS, V. (1994): The economic history of Latin America since independence. New York: Press Syndicate of the University of Cambridge.

CEPAL (2014): Panorama económico y social de América Latina. Publicación de Naciones Unidas, Santiago.

Coloma, F. \& Rojas, P. (2000): Evolución del mercado laboral en Chile, reformas y resultados. En F. LARRAín, \& R. VeRgaRA, La Transformación económica de Chile (págs. 491-540). Santiago: Centro de Estudios Públicos.

CortÁzAr, R. (1993): Política laboral en el Chile democrático: Avances y desafíos en los noventa. Santiago: Ediciones Dolmen.

Daher, A. (1989): Neoliberalismo Urbano en Chile. Ensayo. Centro de Estudios Públicos.

Duccl, M. E. (1997): "Chile: el lado obscuro de una política de vivienda exitosa". Revista Latinoamericana de Estudios Urbano Regionales, 23(69), 99.

EDWARDS, S. (1997): Crisis y reforma en América Latina. Del desconsuelo a la esperanza. Buenos Aires: Emecé Editores.

- (2001): "Veinticinco Años de Inflación y Estabilización en Chile (1973-1998)." En F. LARRAIN \& R. Vergara, La transformación económica de Chile. Santiago: Centro de Estudios Públicos.

FAINSTEIN, S. (2001): "Competitiveness, cohesion and governance: a review of the literature." Cities. New Brunswick: Rutgers University.

FRENCH-DAVIS, R. (2008): Chile entre el neoliberalismo y el crecimiento con equidad. Reformas y políticas económicas desde 1973. Santiago: Comunicaciones Noreste. 
GoRDON, I. (2005): Labour market integration to enhance social cohesion. Paper presented at the OECD International Conference Sustainable Cities: Linking competitiveness with social cohesion. Montreal: OECD

HagGaRD, S. \& Kaufman, R. (1992): The politics of economics adjustment: International constraints, distributive conflicts, and the State. Princeton: Princeton University Press.

HaRberger, A. (1979): "Notas sobre los problemas de vivienda y planificación de la ciudad". AUCA, 37: 39-41.

HARVEY, D. (2007): Breve historia del neoliberalismo. Madrid: Akal.

HIDALGo, R. (1999): "Continuidad y cambio en un siglo de vivienda social en Chile (1892-1998). Reflexiones a partir del caso de la ciudad de Santiago". Revista de geografía Norte Grande, 26. Pag 69-77.

- (1999): "La vivienda social en Chile: la acción del Estado en un siglo de planes y programas." Scripta Nova, 45(1)

- (2004): "La vivienda social en Santiago de Chile en la segunda mitad del siglo XX: Actores relevantes y tendencias espaciales." En Santiago en la Globalización ¿una nueva ciudad? $219-241$.

KAZTMAN, R. (2009): "La dimensión espacial de la cohesión social en las grandes ciudades de América Latina. Seminario regional Alcanzando convergencias en la medición de la cohesión social." Santiago: CEPAL.

- \& Retamoso, A. (2005): Segregación espacial, empleo. Revista de la CEPAL, 85, 131.

LaRraín, F. \& Vergara, R. (2001): "Un cuarto de siglo de reformas fiscales". In F. LaRRAín \& R. Vergara, La transformación económica de Chile. Santiago. Centro de Estudios Públicos.

Link, F. \& ValenZuela, F., \& Fuentes, L. (2015): "Segregación, estructura y composición social del territorio metropolitano en Santiago de Chile: Complejidades metodológicas en el análisis de la diferenciación social en el espacio." Revista de geografía Norte Grande, (62), 151-168.

Minvu (1981 a): "Política Nacional de desarrollo Urbano, Chile, 1979". EURE, Vol. VIII, No 22: 9-15.

- (1981 b): "Conceptos básicos para la formulación de la Política Nacional de Desarrollo Urbano. Chile 1979". EURE, Vol. VIII, No 22: 16-28.

- (1985): "Política Nacional de Desarrollo Urbano". División de Desarrollo Urbano.
- (2009): Política Nacional de Desarrollo Urbano. Ciudades sustentables. Documento de Trabajo, División de Desarrollo Urbano. Santiago.

- /PNUD (2012): Hacia una nueva política urbana para Chile. Antecedentes Históricos. LOM Ediciones. Santiago.

Portes, A. \& Roberts, B. (2005): "La ciudad bajo el libre mercado: La urbanización de América Latina durante los años del experimento neoliberal." Working Paper Series. Princeton University.

Programa de las Naciones Unidas para el Desarrollo (PNUD) (2015): Panorama general: Informe sobre desarrollo humano 2015. Trabajo al servicio del desarrollo humano. New York 2015, 48 páginas.

- (2015): Informe sobre desarrollo humano 2014 Sostener el progreso humano: Reducir vulnerabilidades y construir resiliencia. New York 2015, 4243 páginas.

RIVERA, A. (2012): "Historia de la política habitacional en el área metropolitana de Santiago". Revista CIS, Vol. $16 \mathrm{~N}^{\circ} 16$.

Rodriguez, A., \& Sugranyes, A. (2004): "El problema de vivienda de los "con techo"'. EURE, 30(91), 53-65.

SABATINI, F. (2000): "Reforma de los mercados de suelo en Santiago, Chile: efectos sobre los precios de los mercados de la tierra y la segregación residencial". EURE, 77(26); 49-80.

— \& CÁceres, G., \& Cerda, J. (2001): "Segregación residencial en las principales ciudades chilenas: Tendencias de las tres últimas décadas y posibles cursos de acción". EURE, 27(82), 21-42.

SieRralta, C. (2008): Efectos de la segregación re-sidencial socioeconómica en los jóvenes de extracción popular en Santiago de Chile (1992-2002). Tesis para optar al grado de Magister en Desarrollo Urbano. Instituto de Estudios Urbanos y Territoriales. PUC, Santiago

Theodore, N. \& Peck, J. \& Brenner, N. (2009): "Urbanismo neoliberal: la ciudad y el imperio de los mercados". Temas sociales, 66, 1-13.

TUROK, I. (2006): The connections between social cohesion and city competitiveness. In OECD, Competitive Cities in the global economy. Paris: OECD.

WELLER, J. (2000): Reformas económicas, crecimiento y empleo: Los mercados del trabajo en América Latina y el Caribe. Santiago de Chile: FCE.

- (2011): "Panorama de las condiciones de trabajo en América Latina". Nueva Sociedad (232), 32-49. 